\title{
Computational design of a heated PMMA window validated by infrared thermography
}

\author{
Andreas Rühl[D - Stefan Kolling • \\ Volker Mende • Bernd Kiesewetter
}

Received: 24 June 2015 / Accepted: 6 January 2016 / Published online: 3 February 2016

(C) Springer International Publishing Switzerland 2016

\begin{abstract}
The thermal behavior of a heated window made of poly(methyl methacrylate) was investigated. Two specimens with different numbers of heating lines were prepared and tested at an initial temperature of $-20^{\circ} \mathrm{C}$. The experiments were supervised with an infrared camera operating in the mid-wavelength area. An estimation of uncertainty was performed, because infrared measurements at low temperatures may exhibit greater magnitudes in measurement error. Subsequently, the finite element method was used for simulation of the experiments. Boundary conditions, like convection and power dissipation, were initially estimated and subsequently numerically optimized. The convection coefficient and the input power showed to be crucial design parameters of the simulation. Comparison between experiments and simulations showed temperature deviations within some Kelvin for different heating lines, but the average experimental values were in good agreement with simulation results. The presented numerical model may be used henceforth for further cost-efficient parameter studies concerning
\end{abstract}

\footnotetext{
A. Rühl $(\varangle) \cdot$ S. Kolling

Institute of Mechanics and Materials, THM University of Applied Sciences, Wiesenstraße 14, 35390 Gießen, Germany

e-mail: andreas.ruehl@me.thm.de

V. Mende $\cdot$ B. Kiesewetter

Evonik Industries AG, Kirschenallee, 64293 Darmstadt, Germany
}

window and heating line material, heating line geometry and arrangement, and different boundary conditions.

Keywords Thermography · FEM · Heated window · Validation $\cdot$ Automotive $\cdot$ PMMA

\section{Introduction}

Substitution of traditional materials with polymers has by now been common practice for several decades in the automotive industry. Obvious advantages of polymers are low densities and easy formability. Alternatives for glass usually have to exhibit an amorphous structure considering optical properties. Common polymers fulfilling this requirement are polycarbonate (PC) and poly(methyl methacrylate) (PMMA). PMMA, furthermore, shows outstanding weatherability which makes its usage in outside application attractive. The thermal behavior of PMMA distinguishes significantly from that of glass. The thermal conductivity $k$ of conventional soda-lime glass is approximately one magnitude greater than that of PMMA, whereas the heat capacity $c$ of glass is approximately half that of PMMA. Although the application of these polymers is widely spread for example in car headlights, most car windows are still made of glass, because of more complex technical requirements and safety regulations. This shows to be a remarkable potential for further application of amorphous polymers. 
Table 1 Thermal requirements for a heated rear window

\begin{tabular}{lll}
\hline$\theta_{\max }$ & $\Delta \theta_{D}$ & $\Delta \theta_{R}$ \\
$60^{\circ} \mathrm{C}$ & $5^{\circ} \mathrm{C}$ & $13{ }^{\circ} \mathrm{C}$ \\
\hline
\end{tabular}

In particular, the rear window of a passenger car bears great potential for mass reduction by the substitution of glass with PMMA. Nowadays, these rear windows are usually connected to a heating system for defogging and defrosting. Therefore, these windows must fulfill requirements not only regarding the mechanical properties but also the thermal behavior. These requirements vary for different manufacturers and countries, but may be narrowed down to

- a maximum temperature $\theta_{\max }$ that is not allowed to be exceeded considering thermal expansion,

- a maximum temperature difference $\Delta \theta_{D}$ considering thermal stresses and

- a minimum temperature rise $\Delta \theta_{R}$ inside the heating field to guarantee defrosting.

These requirements are given in Table 1 for one manufacturer and are the basis for the conducted investigation. The fulfillment of the given requirements is verified with a thermographic system. Thermography describes the visualization of radiated energy of a surface that reaches a detector, which enables to determine the surface temperature. It is a common tool in various applications, which is summarized for example in Kylili et al. (2014) as well as Meola and Carlomagno (2004).

Measurements at low temperatures, especially below $0{ }^{\circ} \mathrm{C}$, exhibit additional sources of uncertainty. These are caused by the low energy level reaching the detector array, which leads to a low signal-to-noise ratio. Further uncertainties result from freezing air humidity on the surface of the measured object. These layers exhibit an isolating effect as well as a change in the surface emissivity. The validity of the obtained data from such surfaces requires discussion.

To ensure a reliable and cost-efficient development the finite element method (FEM) is a common tool to reduce experiments but has not been found used for this kind of application so far. Therefore, it requires a basic evaluation of the possibilities and limitations of this method by comparison to experimental results coming from IR measurements. The combination of FE simulations validated by IR thermography has been performed for various topics like light emitting diodes (LED) in Hsieh et al. (2011), polymer chain reaction (PCR) chips in El-Ali et al. (2004) or the thermal behavior of a heat exchanger in Bury and Kruczek (2008). In Lee and Vachtsevanos (2002) IR measurements have been found to be used for the detection of defects within the electrical circuit of a heated rear window. However, the thermal behavior of a heated automotive window partly been treated in Rühl et al. (2014) using the FEM in combination with IR measurements is a current topic that has.

\section{Experiments}

The experimental setup is shown in Fig. 1. Here, a PMMA (PLEXIGLAS ${ }^{\circledR}$ ) window of $4 \mathrm{~mm}$ thickness was positioned inside a climate chamber (2) which is able to operate from -45 to $-10^{\circ} \mathrm{C}$. The climate chamber was set to $-20^{\circ} \mathrm{C}$. Heating power was induced electrically with a power supply unit (3). The infrared camera (IRC) (1) was placed at a distance of $2 \mathrm{~m}$ orthogonally to the window. Heat sources like pipes (5) were covered with a non transmissive fabric, which is not shown in Fig. 1. IR images were taken every second and were stored directly in the associated notebook (4). The upper side of the chamber had to be opened during the experiment for the IRC to cover the whole window. Therefore, higher temperatures were to be expected than adjusted in the chamber and additional uncertainties as well as a non-equilibrium temperature state distribution inside the climate chamber had to be accepted.

\subsection{Specimen preparation}

A picture of specimen $\mathrm{A}$ is shown in Fig. 2. It consists of 13 heating lines that are supplied by bus bars on each side. For the given specimen geometry, this leads to a heating line or interspace distance respectively of approximately $36 \mathrm{~mm}$. Specimen B is built up analogously with 24 heating line resulting in an interspace distance of approximately $16 \mathrm{~mm}$. Considering preliminary finite element (FE) calculations, specimen $\mathrm{A}$ is expected to fail the fulfillment of the requirements from Table 1 because of excessive temperature differences within the heating field. In contrast, specimen $\mathrm{B}$ was calculated to match all requirements, which was 


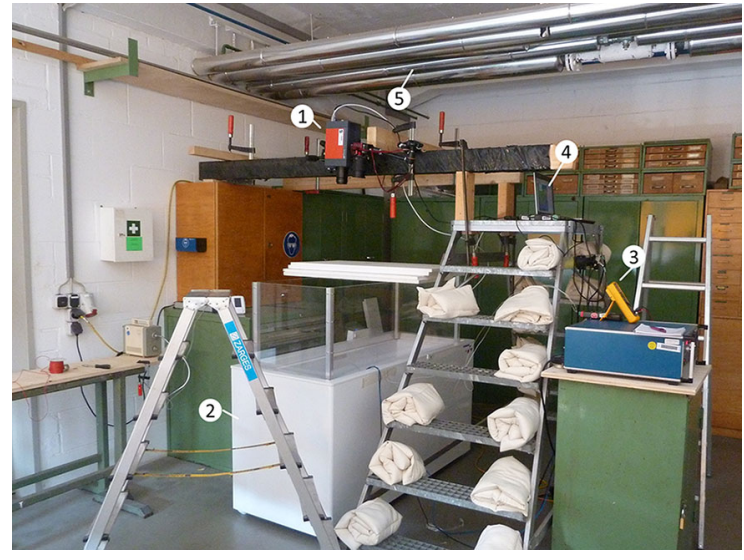

Fig. 1 Experimental setup

affirmed by the experiments. Two thermocouples (TC), as shown in Fig. 2, are used to verify the initial temperature of the IRC. The TC are placed inside the window at approximately half thickness as shown in Fig. 2. For that purpose, the TC were adhered with a onecomponent acrylic-based reactive cement ACRIFIX $^{\circledR}$ 1R0192) inside the specimen. One TC was positioned above one heating line, the other one in the center of the interspace between two heating lines. TC and IRC measurements show good agreement for the initial temperature with deviations lying within the devices uncertainties. However, deviations between TC and IRC began rising during the experiment, which may be caused by different factors. First, the cement used to adhere the TC may lead to significant isolation effects of the measurement spots and TC measurements. Furthermore, the TC exhibit comparatively high time constants for establishing equilibrium temperature with the measured object. Additionally, the TC were placed in the center of the window in thickness direction, whereas the IRC measured the surface temperature only. This makes a direct comparison between IRC and TC for the transient experiment not possible without further investigation of heat fluxes and quantification of isolation effects. However, for the initial temperature, which to be established in a thermodynamic equilibrium, the comparison between both devices is reasonable.

\subsection{Instrumentation}

The IRC camera model used in the present work is the ImageIR $5320 \mathrm{~S}$, fabricated by the company Infratec

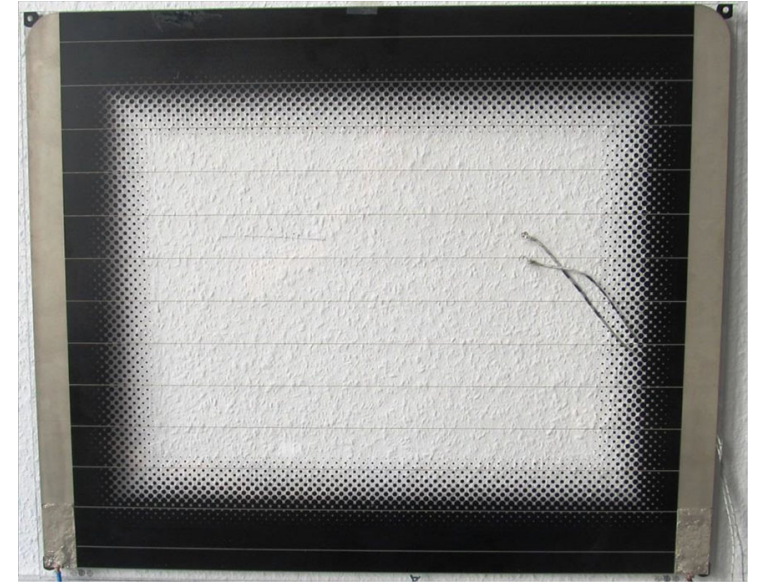

Fig. 2 Specimen A with heating lines and TC

$\mathrm{GmbH}$, and is based on a mid wave (3.7-4.8 $\mu \mathrm{m})$ operating cooled mercury cadmium telluride (MCT) focalplane-array (FPA) detector system. Data acquisition was performed in a full frame mode (320x256IR pixels) with a constant integration time of $1900 \mu \mathrm{s}$. Calibration was performed by the manufacturer to fulfill an uncertainty of $\pm 1{ }^{\circ} \mathrm{C}$ or $\pm 1 \%$ within a temperature range from -30 to $30^{\circ} \mathrm{C}$. The $\mathrm{TC}$ consist of NiCr-Ni (type $\mathrm{K}$ ) and have an uncertainty of $\pm 1.5^{\circ} \mathrm{C}$ within the relevant temperature range. The temperature of the climate chamber can be adjusted within an uncertainty of $\pm 2{ }^{\circ} \mathrm{C}$ for a temperature range between -45 and $-10^{\circ} \mathrm{C}$. However, the real uncertainty may be higher due to inhomogeneous temperature distribution caused by convective turbulences that are most likely additionally intensified due to the open upper side.

\subsection{Uncertainty estimation}

Although the uncertainty of measurement for the IRC is given with $\pm 1{ }^{\circ} \mathrm{C}$ respectively $\pm 1 \%$, the total uncertainty may be significantly higher because of additional error sources at low temperatures. An analysis of possible errors coming from the uncertainty in emissivity and the isolating effects because of an ice layer was performed for the PMMA surface. Measurements of transmissivity and emissivity respectively have not been performed but according to the manufacturer of PMMA an emissivity between 0.9 and 0.95 can be assumed. Ice and snow show emissivities close to 1 , so the error resulting from different emissivities is low for this case, 
especially at these low temperature levels. Yet, isolating effects may lead to major deviations in the calculated temperature compared to the real surface temperature. This makes direct temperature evaluation of ice-covered objects highly questionable without great effort in calculation of thermal behavior of the layer. Consequently, only ice-free windows are discussed in the present work.

The accuracy of IR measurements may be influenced significantly by several sources. As proposed by Minkina and Dudzik (2009) every IR measurement problem should "be treated on an individual basis". Therefore, a simple transfer of uncertainty values from other authors is not necessarily possible due to the uniqueness of every single experimental setup. An error estimation has to be performed that includes all major contributors to the uncertainty in measurement. The manufacturer's instruction gives the equation of the IRC for determination the temperature from radiation reaching the detector by

$$
\begin{gathered}
\theta=(\Phi \varepsilon)^{-1}\left[\frac{I(\Omega, \theta)-\left(1-\tau_{p}\right) \Phi\left(\theta_{p}\right)}{\tau_{p}}\right. \\
\left.-(1-\varepsilon) \Phi\left(\theta_{a m b}\right)\right]
\end{gathered}
$$

with $I$ representing the intensity depending on the solid angle $\Omega$ and the temperature $\theta$ reaching the detector, $\tau_{p}$ and $\theta_{p}$ being the transmissivity and the temperature of the path respectively, $\theta_{r}$ the average ambient temperature of a blackbody being reflected on the measured object, and $\varepsilon$ being the emissivity of the considered surface. $\Phi(\theta)$ represents the calibration curve of the IR system, which gives the intensity as a function of the temperature $\theta$. For a non-participating path medium Eq. (1) can be simplified to

$\theta=(\Phi \varepsilon)^{-1}\left[I(\Omega, \theta)-(1-\varepsilon) \Phi\left(\theta_{a m b}\right)\right]$.

It can be observed that Eq. (2) gives the calculated temperature $\theta$ as a function of the calibration curve $\Phi(\theta)$, the emissivity $\varepsilon$ respectively the transmissivity $\tau$ of the object and the ambient blackbody temperature $\theta_{r}$. Determination of the values are always accompanied by uncertainties. An absolute uncertainty in measurement was calculated consisting of the instrumented error, the error in emissivity of the measured surface and the additional error coming from the measurement at low temperature. The influence of the reflectivity is included in the emissivity uncertainty. The path was assumed as perfectly transmissive, so no influences of humidity or scattering in the path are considered. An absolute value for the error of measurements regarding an uncertainty span in emissivity between 0.90 and 0.95 and an uncertainty span from -10 to $-20^{\circ} \mathrm{C}$ for the ambient radiation temperature was calculated with $\pm 1.4{ }^{\circ} \mathrm{C}$ for the maximum deviations. The lower limit value for the ambient temperature is given by the temperature, which the climate chamber was set to. The upper limit of $-10^{\circ} \mathrm{C}$ is an estimation of the maximum possible average ambient temperature. Added to the system uncertainty of the IRC a total uncertainty of measurement was calculated to be $\pm 2.4^{\circ} \mathrm{C}$.

\subsection{Experimental results}

Experimental results for both specimens are shown for one comparable input power. The experiments were performed with the heating lines on the bottom side. Figure 3 shows an IR image of specimen A after 10 min of heating with $130 \mathrm{~W}$ input power. Thermal contours of the heating lines are clearly visible. Furthermore, the bus bars on the left and right side show a significant increase in temperature. This indicates a loss of input power before reaching the heating lines. The origin of this could not be fully resolved, but is most likely the result of a defective electrical contact, which leads to a localized heating. Figure 4 shows the related temperature-time distribution for specimen A. The displayed data represent average temperatures along the evaluation lines shown in Fig. 3 for the curves depicted as "heating lines". These lines are positioned on the opposite site of the heating lines in order to capture the maximum temperature on the top side of the window. Analogously, curves depicted as "interspace" represent average temperatures in the center between two heating lines in order to measure the greatest possible temperature difference within the heating field. For both heating lines and interspaces, 3 representative curves are labeled with the associated line or interspace number shown in Fig. 3. These curves are the maximum, minimum and one value close to average in the temperature time distribution. The measurements show a significant deviation of the interspace temperature to the heating lines temperature and therefore an exceeding of the allowed maximum temperature difference, which is given in Table 1. Furthermore, a major devia- 

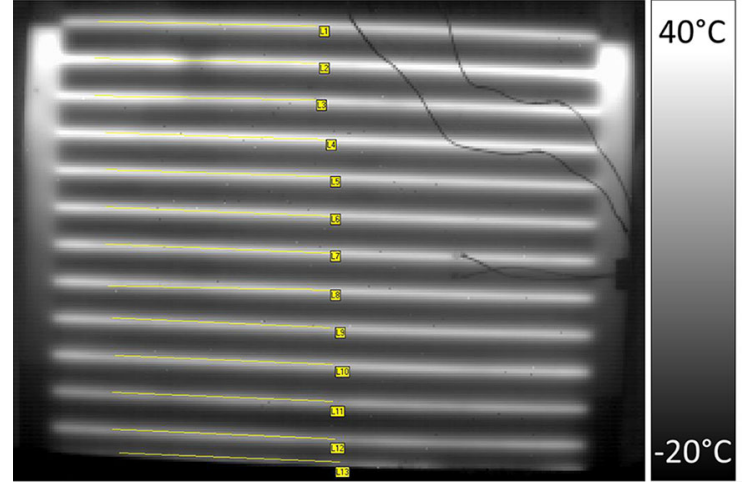

Fig. 3 Specimen A: IR image after $10 \mathrm{~min}$ at $130 \mathrm{~W}$ input power

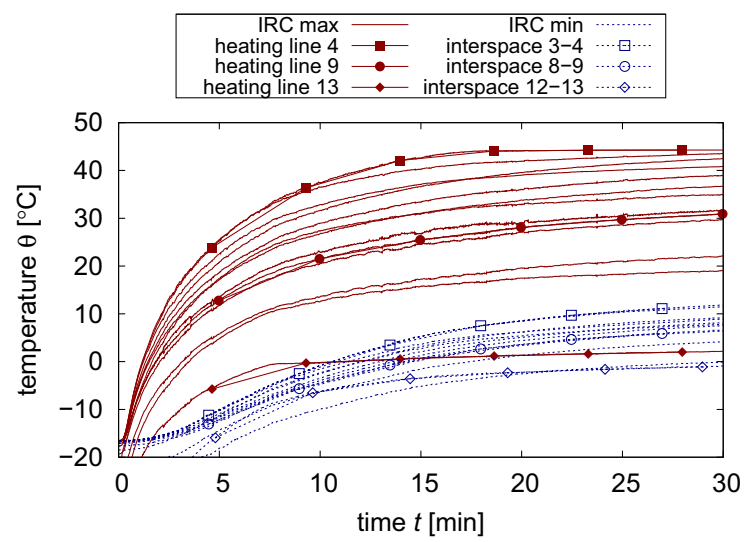

Fig. 4 Specimen A: experimental results for minimum and maximum temperature measured with IRC for one heating line and the neighboring interspace

tion between single heating lines can be observed and a very inhomogeneous temperature field is apparent. Temperatures significantly above $30^{\circ} \mathrm{C}$ are outside the calibration curve and their real value may therefore be higher than calculated.

Based on the evaluation of specimen A, a second specimen was produced with respect to carefully installed electrical contacts and a smaller heating line distance. Figure 5 shows the temperature measurement for specimen B with analogous measurement technique as applied for specimen A. A similar input power as used for specimen A is used and a more homogeneous temperature distribution and smaller temperature differences are reached. Furthermore, no significant heating is observed at the bus bars and therefore smaller losses than for specimen A can be assumed. Specimen B fulfills all given requirements from Table 1 and is therefore aim of the numerical investigation performed

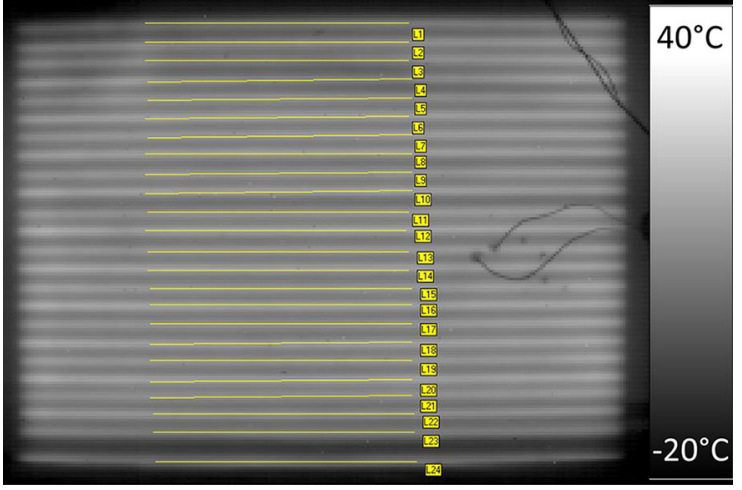

Fig. 5 Specimen B: IR image after $10 \mathrm{~min}$ at $125 \mathrm{~W}$ input power

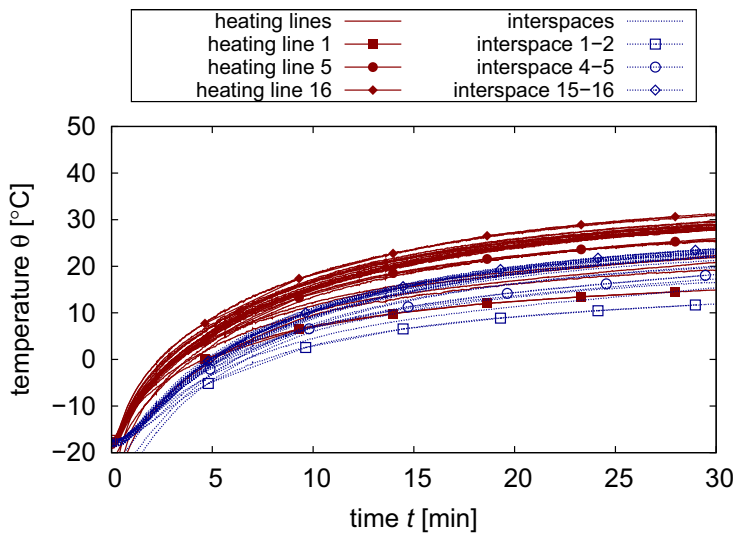

Fig. 6 Specimen B: experimental results for minimum and maximum temperature measured with IRC for one heating line and the neighboring interspace

subsequently. However, some inhomogeneities in the temperature distribution could still be observed. These are shown in Fig. 7, where the temperature distribution of all heating lines is shown. For the same input power, temperatures between approximately 14.5 and $18.5^{\circ} \mathrm{C}$ were noticed.

\section{Numerical calculations}

The specimen preparation showed to be very exhaustive in time and resources. A numerical method in order to estimate the amount of heating lines and input power of upcoming specimen for further development is therefore desirable. In the present work, the FEM is used to calculate the problem. In this method the considered window and heating lines are divided into a finite number of elements that approximate the real geometry. 


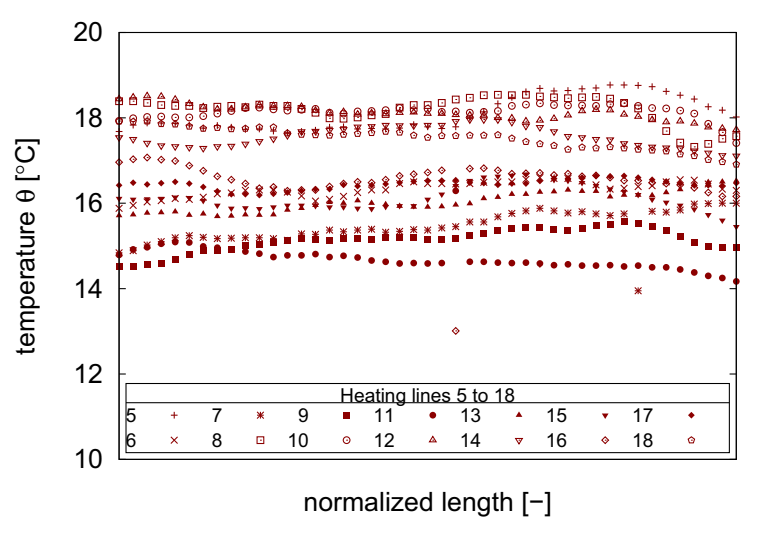

Fig. 7 Temperature bandwidth for heating lines of specimen B at $10 \mathrm{~min}$

Temperatures can be calculated on an element level by solving Fourier's law, which is given in Lienhard (2013) by

$\mathbf{q}=-k \nabla \theta$

with $\mathbf{q}$ representing the heat flux and $k$ the thermal conductivity. With given boundary conditions a functional $\Pi$ can be obtained, which is used by Bathe (2002) to express the principle of virtual temperatures with the requirement of stationarity by

$$
\begin{aligned}
& \int_{V} \delta \theta_{, i}{ }^{T} k_{i j} \theta_{, j} \mathrm{~d} V=\int_{V} \delta \theta \dot{q}^{B} \mathrm{~d} V+\int_{S_{q}} \delta \theta^{S} \dot{q}^{S} \mathrm{~d} S \\
& +\sum_{i} \delta \theta^{i} Q^{i} .
\end{aligned}
$$

Here, temperatures with a $\delta$-prefix are virtual values. Thermal conductivity is expressed by a second-order tensor. Indices $B$ and $S$ stand for a heat source and incoming heat flux density respectively. Integration of the incoming heat flux density is performed with respect to the boundary of the continuum $S_{q}$. For a linear transient calculation a time step $\Delta t$ is introduced for which at a time of $t+\Delta t$ the principle of virtual temperatures is calculated according to Bathe (2002) by

$$
\begin{gathered}
\int_{V} \delta \theta^{T}(\rho c)^{t+\Delta t} \dot{\theta}^{t+\Delta t} \mathrm{~d} V+\int_{V} \delta \theta_{, i}^{T} k_{i j}^{t+\Delta t} \delta \theta_{, j}^{t+\Delta t} \mathrm{~d} V \\
=Q^{t+\Delta t}+\int_{S_{c}} \delta \theta^{S} h^{t+\Delta t}\left(\theta_{e}^{t+\Delta t}-\theta^{S t+\Delta T}\right) \mathrm{d} S \\
\quad+\int_{S_{r}} \delta \theta^{S t+\Delta t} \kappa\left(\theta_{r}^{t+\Delta t}-\theta^{S t+\Delta t}\right) \mathrm{d} S
\end{gathered}
$$

and can be solved for the current state temperature $\theta^{t+\Delta t}$. With mass density $\rho$ and heat capacity $c$ the increase of internal energy is included. The term $Q^{t+\Delta t}$ represents external heat sources entering the system, indices $c$ and $r$ stand for the convective and radiative terms respectively. The full model is assembled by connecting the single elements in a matrix. The temperature $\theta^{S}, \theta_{e}$ and $\theta_{r}$ refer to the surface, environmental and ambient temperature. Discretization to finite elements is performed and temperatures are calculated by solving Eq. (5) numerically.

The time step $\Delta t$ may be selected freely but is crucial for the solver to find the equilibrium. By choosing a time step of 1 second, the calculation interval is adjusted to the data acquisition rate of the IRC. With this time step, no instabilities in equilibrium determination were found and the time step remained at its maximum value at all times. The problem investigated in the present work contains furthermore contact modeling between heating line elements and the window elements which additionally increases complexity of the numerical model. For time efficient calculation the commercial solver LS-DYNA, version 971 R7.0.0 in double precision and single memory processing (SMP) mode, is used for a fully implicit calculation. The thermal time step of the solution was modeled to be variable with possible time step reduction if the equilibrium criteria are not fulfilled.

\subsection{FE modeling}

A reduced FE model was set up in order to use the model's symmetries in the simulation and is shown in Fig. 8. Both window and heating lines were modeled with solid elements. The contact between heating lines and window is modeled as perfectly closed at all times.

For the heat, which is dispersed by convection, the ambient temperature, which is assumed in paragraph 2.3 to be between -20 and $-10^{\circ} \mathrm{C}$, and the coefficient of convection for the upper and bottom side of the window had to be specified. To simplify the model, heat conduction to the periphery is modeled within this coefficient of convection. The heat flux, which is based on the electrical input power used in the experiment, was multiplied with a constant scale factor in order to take into account power losses before reaching the heating lines. Additionally, experiments showed slight deviations in input power due to changes in electrical 
Fig. 8 Reduced FE model

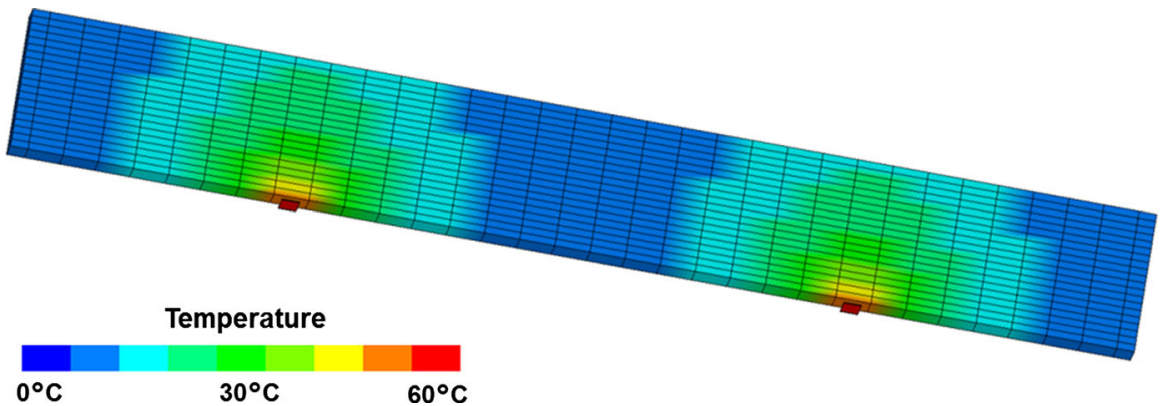

resistance with rising temperature, which was modeled by using time-dependent tabulated values for the input power obtained from the experiment.

The initial temperature is calculated as an average value of all heating lines and interspaces and the emissivity of PMMA was set to 0.92 . Taking into account the uncertainty in power loss and convection, the values input power scale factor, ambient temperature and the two coefficients of convection were assumed to be within a certain range and had to be determined to obtain one final set of parameters. This was performed by numerical optimization using the commercial software LS-OPT. Here, the results of the simulations are compared and adjusted to average experimental temperature-time curves for the heating lines and the interspace values from Fig. 6 using the least-square fit method. Final values for the thermal boundary conditions are summarized in Table 2.

\subsection{Material modeling}

A thermal isotropic material model was used for PMMA and the heating lines. Values for thermal conductivity $k$ and heat capacity $c$ were obtained by Evonik Industries AG and are shown in Fig. 9. Thermal conductivity was determined using regulations from DIN 52612-1, heat capacity determination followed the regulation of DIN EN ISO 11357-4. For room temperature, these results are published in Evonik (2013). Here, a significant change of the heat capacity can be observed, while thermal conductivity only performs small deviation for a large temperature range. For a temperature range of $-20^{\circ} \mathrm{C}$ to approximately $60^{\circ} \mathrm{C}$ a change in heat capacity of approximately $20 \%$ is to be expected. Thermal properties of PMMA have already been investigated several times for example by Assael et al. (2005) or Sargsyan et al. (2007). Results from both authors
Table 2 Thermal coefficients used in FE-simulation

\begin{tabular}{ll}
\hline Upper side & \\
Emissivity & 0.92 \\
Coefficient of convection & $2.58 \mathrm{~W} /\left(\mathrm{m}^{2} \mathrm{~K}\right)$ \\
Ambient temperature & $-15.7^{\circ} \mathrm{C}$ \\
Bottom side & \\
Emissivity & 0.92 \\
Coefficient of convection & $1.0 \mathrm{~W} /\left(\mathrm{m}^{2} \mathrm{~K}\right)$ \\
Ambient temperature & $-15.7^{\circ} \mathrm{C}$ \\
Heating lines & \\
Initial heat flux & $19.59 \mathrm{~mW} / \mathrm{mm}^{2}$ \\
Scale factor & 0.91 \\
\hline
\end{tabular}

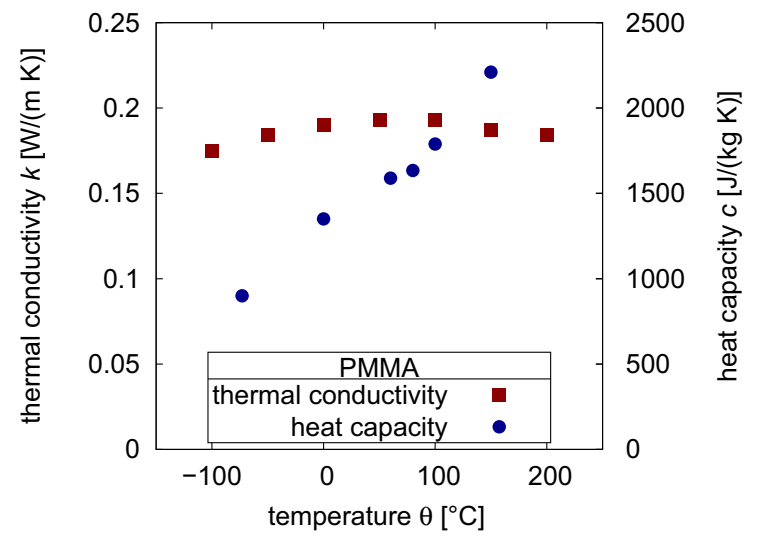

Fig. 9 Thermal conductivity $k$ and heat capacity $c$ for PMMA

show to be in good agreement with the values of the present work. In the simulation, thermal conductivity $k$ and heat capacity $c$ are used with a load curve respecting temperature dependency.

For the heating lines, no measurement of thermal properties were performed. Therefore, constant values given in Howell et al. (2011) for silver were used, which approximates the heating line material. 
Fig. 10 Topography of temperature distribution along 6 top-down lines for experiment and simulation

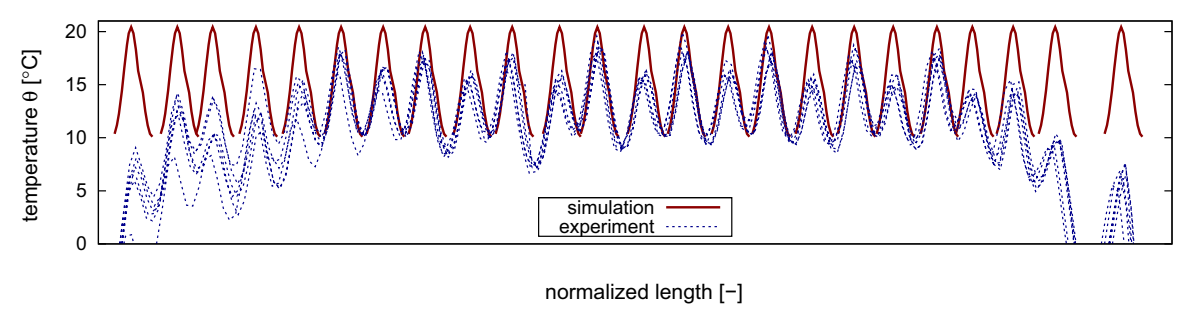

\subsection{Simulation results and discussion}

Figure 10 shows the temperature topography comparison between simulation and experiment at a time of 10 min. The temperature distribution along 6 lines, which are drawn from top down on the window are shown for a time of $10 \mathrm{~min}$. The interspaces in the simulation curve result from deviations in the heating line distance of the experiments. Here, the peak value of each heating line was used as a reference for offsetting the simulation. It is clearly visible that the simulation overestimates the temperature level as well as the peak temperatures (above the heating lines) towards the edges of the window. At the center of the window simulation represents the topography well for most heating lines. A small overestimation of the experiment can be observed for every heating line.

Further discrepancies between simulation and experiment are caused by several factors. First, an inhomogeneous temperature distribution on the window surface is visible even before starting the experiment. This is due to the modeling with a constant initial temperature. Furthermore, the convection is neither a constant value for time or location nor was it experimentally determined. For the optimized coefficients of convection significant deviations to the real values are possible. As a third reason, the loss of power, which is approximated with an optimized scale factor, has great influence on the simulation results.

In Fig. 3, a distinct heat generation can be observed at the bus bars that still contributes to the heating of the window. With respect to the significant inhomogeneities in temperature distribution observable in Figs. 3 and 5, only heating lines close to the average value are approximated by the simulation. Therefore, a central conclusion drawn from these results is the requirement of improving the experimental setup primarily with respect to homogenization of the temperature distribution.

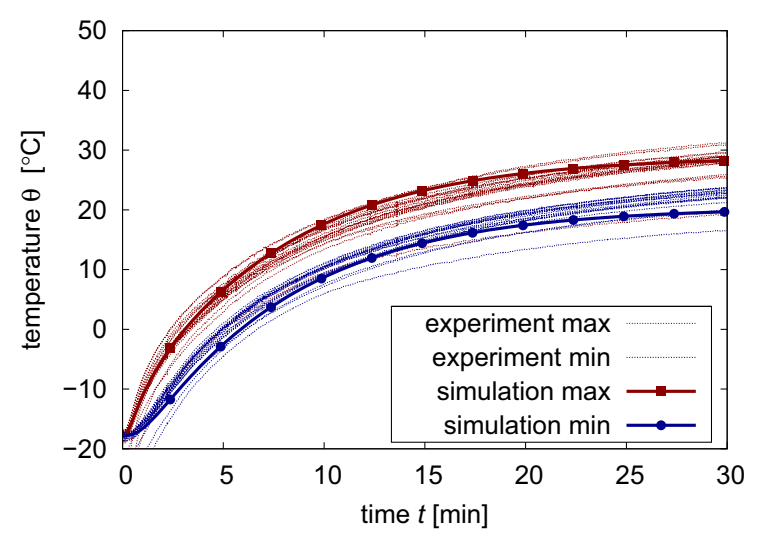

Fig. 11 Comparison of simulation and experiment for minimum and maximum surface temperature

Figure 11 shows the final results of the simulation of the minimum and maximum temperature in comparison to the temperatures of the heating lines and the interspaces. Here, the heating lines 1-4 and 19-24 are neglected for showing little temperature rise. This may have been caused by insufficient conduction of the input power to both ends at the window. The initial temperature of the simulation was idealized to be the same for minimum and maximum. Simulation shows to be within the range of scattering of the heating lines and the interspaces. This shows that the simulation is able to reproduce the experimental results well. Note that simulation slightly overestimates the experimental values at a time of $10 \mathrm{~min}$, which corresponds to overestimation observed for the simulation in Fig. 10.

To sum up the simulation results, the presented model is able to predict the average thermal behavior well. Though it lacks in a local analysis, especially when experimental values show significant inhomogeneities. Modeling of inhomogeneities would require additional effort and a significant complication of the numerical model. Against this background, the presented model appears to be in accordance to good engi- 
neering practice and as a useful tool for the thermal outlaying of windows with heating lines.

\section{Summary}

Experimental and numerical investigation regarding the thermal behavior of a heated PMMA window have been performed. Two specimens were manufactured and supervised with an infrared camera. The low ambient temperature of $-20^{\circ} \mathrm{C}$ lead to additional uncertainties of the IR measurement but results showed to be reliable although a greater range of uncertainty had to be accepted. Thermal behavior of iced windows were also investigated but showed too high deviations from each other mainly caused by the ice layer. The ice layer distorts the temperature determination of the window significantly, which made quantitative measurements only trustworthy for ice-free areas. Subsequently, a feasibility study was performed using the finite element method in order to reduce the experimental effort for future developments. Some thermal values like convection, heating line material properties and input power had to be estimated. A numerical optimization adjusted the parameters of the simulation in a good agreement to the experimental results. This validated finite element model can now be used in engineering practice for design and dimensioning of heated PMMA windows.

\section{Compliance with ethical standards}

Conflict of Interest On behalf of all authors, the corresponding author states that there is no conflict of interest.

\section{References}

Assael, M., Botsios, S., Gialou, K., Metaxa, I.: Thermal conductivity of polymethyl methacrylate (pmma) and borosilicate crown glass BK7. Int. J. Thermophys. 26(5), 1595-1605 (2005)
Bathe, K.J.: Finite-elemente-methoden, vol. 2. Springer, Berlin (2002)

Bury, T., Kruczek, T.: Application of infrared thermography for validation of numerical analyses results of a finned crossflow heat exchanger with non uniform flow of the agents. In: Proceedings 9th International Conference Quantity Infrared Thermography, pp. 291-298 (2008)

El-Ali, J., Perch-Nielsen, I.R., Poulsen, C.R., Bang, D.D., Telleman, P., Wolff, A.: Simulation and experimental validation of a SU-8 based PCR thermocycler chip with integrated heaters and temperature sensor. Sensor Actuator A 110(1), 3-10 (2004)

Evonik Industries AG: Plexiglas GS / XT Technical information. www.evonik.com (2013)

Howell, J.R., Siegel, R., Menguc, M.P.: Thermal Radiation Heat Transfer. CRC Press, (2011)

Hsieh, J.C., Lin, D.T., Cheng, C.H.: Optimization of thermal management by integration of an SCGM, a finite-element method, and an experiment on a high-power LED array. IEEE Trans. Electron Devices 58(4), 1141-1148 (2011)

Kylili, A., Fokaides, P.A., Christou, P., Kalogirou, S.A.: Infrared thermography (IRT) applications for building diagnostics: a review. Appl. Energ. 134, 531-549 (2014)

Lee, S., Vachtsevanos, G.: An application of rough set theory to defect detection of automotive glass. Math. Comput. Simulat. 60(3), 225-231 (2002)

Lienhard, J.H.: A Heat Transfer Textbook. Courier Corporation, (2013)

Meola, C., Carlomagno, G.M.: Recent advances in the use of infrared thermography. Meas. Sci. Technol. 15(9), R27 (2004)

Minkina, W., Dudzik, S.: Infrared Thermography: errors and uncertainties. Wiley (2009)

Rühl, A., Kolling, S., Mende, V., Kiesewetter, B.: Thermal simulation of heated PMMA rear windows. In: Proceedings of the 13th LS-DYNA Forum, Bamberg, Germany (2014)

Sargsyan, A., Tonoyan, A., Davtyan, S., Schick, C.: The amount of immobilized polymer in pmma $\mathrm{SiO}_{2}$ nanocomposites determined from calorimetric data. Euro. Polym. J. 43(8), 3113-3127 (2007) 